\title{
Projets parentaux des femmes vivant avec le VIH/ sida à l'ère des thérapies antirétrovirales
}

\author{
Isabelle HEARD ${ }^{1}$, Rémi SITTA, France LERT ${ }^{2}$ et le groupe d'étude Anrs-Vespa ${ }^{3}$
}

\section{Résumé}

L'évolution, au cours de la dernière décennie, de la structure démographique et épidémiologique de l'épidémie par le $\mathrm{VIH} /$ sida amène à faire le point sur les projets parentaux des personnes atteintes. En effet, dans le contexte actuel, grâce aux traitements antirétroviraux, un contrôle efficace de l'infection chez les parents potentiels vivant avec le VIH est assuré, de même qu'un fort taux de réduction du risque de transmission du virus de la mère à l'enfant.

L'étude présentée ici concerne le désir d'enfant des femmes en âge de reproduction, ayant participé à l'enquête Anrs-Vespa, en fonction de l'origine ethnique, du mode de vie, de facteurs culturels et de santé (état immunovirologique, traitement).

Cinq cent cinquante cinq femmes se déclarant hétérosexuelles et qui n'avaient pas d'antécédent connu d'infertilité ont été incluses dans l'analyse. Parmi elles, $33 \%$ ont rapporté le désir d'avoir des enfants. L'âge moyen des femmes retenues dans l'analyse est de 36 ans (31-40). L'Afrique sub-saharienne est le pays d'origine de $30 \%$ d'entre elles.

Chez les femmes séropositives, les déterminants d'un projet d'enfant mis en évidence dans cette étude comprennent d'une part, des facteurs classiques comme le jeune âge, le fait de vivre en couple stable et de ne pas être déjà mère et, d'autre part, des facteurs culturels comme I'origine ethnique (naissance au Maghreb ou en Afrique sub-saharienne). Par ailleurs, les couples sont plus susceptibles de s'engager dans un projet parental si au moins l'un d'eux n'est pas infecté par le VIH. L'état de santé par rapport au VIH/sida n'est pas associé au projet parental.

Au vu de ces résultats, il semble que la problématique de la parentalité devrait dorénavant être mieux prise en compte au cours de la prise en charge médicale de la séropositivité.

Mots clés : désir d'enfant, VIH, déterminants sociodémographiques, comportements.

L'infection par le VIH concerne de plus en plus de femmes dans le monde. La plupart sont jeunes, en âge de faire des enfants et

\footnotetext{
Unité de biologie de la reproduction, groupe hospitalier Pitié-Salpêtrière, Université Paris VI, Paris France.

2 Unité Inserm 687-IFR 69, Saint-Maurice, France.

Groupe Anrs-Vespa: AD Bouhnik (unité Inserm 379/0bservatoire régional de la santé de la région PACA), R Dray-Spira (unité Inserm 687-IFR 69), J Fagnani (CNRS-UMR Matisse), I Heard (groupe hospitalier Pitié-Salpêtrière), F Lert (unité Inserm 687-IFR 69), Y Obadia (Observatoire régionalde la santé PACA/unité Inserm 379), P Peretti-Watel (Observatoire régional de la santé PACA/unité Inserm 379), J Pierret (CERMES-unité Inserm 750-CNRS UMR 8559), B Riandey (Ined), MA Schiltz (CERMES-unité Inserm 750-CNRS UMR 8559), R Sitta (unité Inserm 687-IFR69), B Spire (unité Inserm 379/ORS PACA).
}

doivent faire face à des choix difficiles en matière de reproduction. Dans les premières années de l'épidémie, peu d'attention était portée à tout ce qui concerne la reproduction pour les couples vivant avec le $\mathrm{VIH} /$ sida, du fait du fort taux de mortalité des adultes et des enfants, et du risque élevé de transmission du virus de la mère à l'enfant. Mais aujourd'hui, les désirs de reproduction des personnes vivant avec le $\mathrm{VIH} /$ sida doivent être pris en compte [1-3]. En effet, dans les pays développés, la mortalité et la morbidité liées à l'infection par le VIH ont beaucoup diminué ces dix dernière décennies, avec l'arrivée des traitements antirétroviraux hautement efficaces, la trithérapie [4]. Une meilleure connaissance des mécanismes de la transmission du virus de la mère à l'enfant a également permis d'abaisser fortement le taux de transmission du VIH. La prise en charge de la grossesse, de l'accouchement et du nourrisson avec des traitements et des techniques adaptées s'accompagne maintenant d'un taux de transmission aux alentours de $1 \%$ [5]. Cependant, d'autres facteurs liés au fait d'être séropositif peuvent intervenir dans la décision d'avoir ou non un enfant. La stigmatisation à l'égard des personnes vivant avec le VIH/sida est fréquente. Les conditions de vie sont parfois difficiles et le manque de ressources n'est pas rare chez les femmes séropositives. Beaucoup appartiennent à des minorités ethniques, sont récemment immigrées et encore mal intégrées à la société française. C'est pourtant parfois dans ces situations difficiles que le désir d'enfant et d'épanouissement en tant que parent s'exprime avec force.

L'objectif de cet article est de décrire les facteurs sociodémographiques et de santé associés au désir d'enfant chez des femmes séropositives pour le $\mathrm{VIH}$, en âge d'avoir des enfants.

\section{Méthodologie}

L'échantillon constitué pour notre étude est établi à partir des patients de l'enquête Anrs-Vespa. Celle-ci, menée en 2003, a inclus 2932 patients atteints d'une infection à $\mathrm{VIH}$, suivis dans des services hospitaliers. Pour une présentation détaillée de cette enquête, voir l'encadré page 215 [6].

Nos analyses ont été restreintes aux participantes en âge de reproduction (âgées de 18 à 44 ans), se définissant elles-mêmes comme hétérosexuelles et ne se connaissant pas d'antécédents de stérilité $(n=555)$. 
Le " désir d'enfant " a été défini à partir de réponses aux questions suivantes : 1) «Êtes-vous enceinte ? "; 2) "Cherchez-vous actuellement à avoir un enfant? "; 3) «Avez-vous le désir d'avoir un enfant ?"; 4) "Avez-vous le désir d'avoir un enfant peutêtre plus tard?"; (5) "Vous ne souhaitez pas avoir d'enfant". Si la femme répondait par l'affirmative à la question (1), (2) ou (3), elle était considérée comme ayant un désir d'enfant. Si elle répondait oui à la question (4) ou (5), elle était considérée comme n'ayant pas de désir d'enfant. Ce regroupement a été effectué en concordance avec une étude nationale française concernant la fécondité des femmes en France [7].

Notre étude a consisté à étudier les associations entre différents facteurs sociodémographiques, comportementaux et de santé, et le désir d'avoir un enfant. Les données ont été pondérées de manière à tenir compte de l'échantillonnage, des biais de participation et de la fréquence des consultations qui influençaient la probabilité d'être tirée au sort pour l'enquête. Les estimations de variance calculées ont intégré cette pondération, ainsi que le plan de sondage.

En analyses univariée et multivariée, les facteurs suivants ont été testés comme facteurs associés à un désir d'enfant : l'âge, le pays de naissance, le fait d'avoir un emploi, le niveau d'éducation, le fait d'avoir déjà un enfant, l'année de diagnostic de la séropositivité, le fait d'être en traitement pour le VIH, d'avoir un bon contrôle de l'infection à VIH (chiffre de CD4 supérieur à 200 cellules $/ \mathrm{mm}^{3}$, charge virale inférieure à 400 copies $/ \mathrm{ml}$ ), un antécédent de toxicomanie intraveineuse, un stade sida " $\mathrm{C}$ " et le mode de transmission du VIH. La relation de couple a été caractérisée en fonction du fait d'avoir un partenaire régulier, de son statut par rapport au VIH et d'avoir eu ou non des partenaires occasionnels dans les 12 mois précédant l'entretien. Différentes situations de couple ont été répertoriées : en couple avec un partenaire régulier séropositif avec ou sans partenaires occasionnels; en couple avec un partenaire régulier séronégatif ou de statut VIH inconnu sans partenaires occasionnels; en couple avec un partenaire régulier séronégatif ou de statut $\mathrm{VIH}$ inconnu avec partenaires occasionnels ; seulement des partenaires occasionnels ; pas d'activité sexuelle dans les douze derniers mois.

Les analyses univariées sont présentées en utilisant le pourcentage pondéré de désir d'enfant. Tous les facteurs ont été considérés dans l'analyse multivariée excepté le mode d'infection car fortement corrélé aux autres facteurs.

\section{Résultats}

Parmi les 794 femmes de l'étude Vespa, 216 ont été exclues de l'analyse car âgées de plus de 44 ans, quatre femmes ont été exclues car elles se définissaient comme homosexuelles et 19 femmes ont été exclues car elles rapportaient une infertilité.

Le tableau 1 montre les caractéristiques des 555 femmes en âge de reproduction finalement retenues dans l'analyse. L'âge
Tableau 1

Caractéristiques des femmes incluses $(n=555)$

\begin{tabular}{|c|c|c|}
\hline & n & $\%$ pondéré \\
\hline \multicolumn{3}{|c|}{ Année de diagnostic de la séropositivité VIH/sida } \\
\hline $1980-1987$ & 87 & 15,1 \\
\hline $1988-1990$ & 87 & 15,0 \\
\hline $1991-1993$ & 86 & 14,9 \\
\hline 1994-1996 & 71 & 13,1 \\
\hline $1997-1999$ & 91 & 18,3 \\
\hline $2000-2003$ & 133 & 23,6 \\
\hline \multicolumn{3}{|l|}{ Âge à l'enquête Vespa } \\
\hline$<30$ ans & 88 & 15,7 \\
\hline $30-34$ ans & 121 & 23,6 \\
\hline $35-39$ ans & 174 & 30,5 \\
\hline $40-44$ ans & 172 & 30,2 \\
\hline \multicolumn{3}{|l|}{ Activité } \\
\hline Emploi & 245 & 48,2 \\
\hline Chồmage & 132 & 24,0 \\
\hline Inactivité & 178 & 27,8 \\
\hline \multicolumn{3}{|l|}{ Niveau d'études } \\
\hline Collège & 162 & 28,5 \\
\hline CAP, BEP & 125 & 22,0 \\
\hline Niveau lycée & 153 & 27,7 \\
\hline Cycle supérieur & 115 & 21,9 \\
\hline \multicolumn{3}{|l|}{ A des enfants } \\
\hline Oui & 246 & 45,0 \\
\hline Non & 309 & 55,0 \\
\hline \multicolumn{3}{|l|}{ Situation de couple } \\
\hline Pas d'activité sexuelle dans l'année & 111 & 18,5 \\
\hline Couple séroconcordant* & 87 & 16,6 \\
\hline Couple sérodiscordant exclusif** & 235 & 43,6 \\
\hline Couple sérodiscordant non exclusif ${ }^{* * *}$ & 35 & 6,1 \\
\hline Partenaires occasionnels uniquement & 87 & 15,2 \\
\hline \multicolumn{3}{|l|}{ Pays de naissance } \\
\hline France/Europe & 355 & 62,6 \\
\hline Afrique sub-saharienne & 164 & 30,3 \\
\hline Maghreb & 18 & 3,5 \\
\hline Autres & 18 & 3,6 \\
\hline \multicolumn{3}{|l|}{ Toxicomanie intraveineuse } \\
\hline Oui & 122 & 20,1 \\
\hline Non & 433 & 80,0 \\
\hline \multicolumn{3}{|l|}{ Stade sida } \\
\hline Oui & 102 & 15,7 \\
\hline Non & 453 & 84,3 \\
\hline \multicolumn{3}{|l|}{ Traitement antirétroviral } \\
\hline Oui & 434 & 76,0 \\
\hline Non & 121 & 24,0 \\
\hline \multicolumn{3}{|l|}{ Bon contrôle de l'infection à VIH } \\
\hline Oui & 318 & 58,3 \\
\hline Non & 237 & 41,7 \\
\hline \multicolumn{3}{|l|}{ Mode de transmission } \\
\hline Toxicomanie intraveineuse & 103 & 16,5 \\
\hline Hétérosexuelle & 421 & 78,8 \\
\hline Autre & 31 & 4,7 \\
\hline
\end{tabular}

- Partenaire masculin séropositif pour le VIH, sans partenaires occasionnels.

* Partenaire masculin séronégatif pour le VIH, sans partenaires occasionnels.

*. Partenaire masculin séronégatif pour le VIH, avec partenaires occasionnels. 
médian était de 36 ans (31-40). La majorité des femmes étaient nées en France ou en Europe ( $63 \%$ ), alors que $30 \%$ d'entre elles étaient nées en Afrique sub-saharienne. Près d'une femme sur deux avait une activité professionnelle au moment de l'étude. Les femmes avaient été infectées par contact sexuel dans $79 \%$ des cas et par injection intraveineuse de drogue dans $17 \%$ des cas. Près d'une femme sur deux ( $45 \%$ ) était mère.

En ce qui concerne leur vie sexuelle, une femme sur deux (51 \%) avait un partenaire depuis au moins six mois, celui-ci étant le plus souvent séronégatif pour le VIH (76 \% des cas). Une femme sur cinq environ (19\%) n'avait pas eu de rapport sexuel dans l'année précédente, $15 \%$ n'avaient que des partenaires occasionnels.

Les trois-quarts des femmes étaient sous traitement antirétroviral et $58 \%$ avaient de bons paramètres biologiques de leur infection (CD4 $>200$ cellules $/ \mathrm{mm}^{3}$ et charge virale $<400$ copies $/ \mathrm{ml}$ ).

Une femme sur trois (33\%) rapportait être enceinte ou avoir un désir d'enfant : $3 \%$ étaient enceintes, $6 \%$ essayaient d'avoir un enfant et $23 \%$ disaient vouloir avoir un enfant dans un avenir proche.

L'analyse univariée des facteurs sociodémographiques et comportementaux montre que le fait d'être jeune (moins de 30 ans), d'être dans une relation stable avec un partenaire séronégatif pour le VIH, d'être née en Afrique du Nord ou sub-saharienne, de ne jamais avoir pris de drogue intraveineuse, étaient des facteurs significativement associés à un désir d'enfant (tableau 2). Le désir d'enfant était plus fréquent chez les femmes dont la séropositivité avait été découverte après 1995 que chez celles dont le diagnostic était antérieur. II était moins fréquent chez les femmes qui étaient déjà mères que chez celles qui n'avaient pas d'enfant. Les femmes sans emploi exprimaient moins souvent un désir d'enfant que celles qui avaient une activité professionnelle.

Aucune liaison significative n'a été mise en évidence entre le stade évolutif de la maladie VIH/sida, le fait d'être sous traitement antirétroviral ou le statut vis-à-vis de la maladie et le désir d'enfant.

L'utilisation de modèles de régression logistique a permis de montrer que le désir d'enfant était deux fois plus fréquent chez les femmes âgées de moins de 30 ans que chez celles de 30 à 34 ans (odds ratio $(O R)=2,01)$; intervalle de confiance $(I C)$ à $95 \%$ [1,03-3,92] (tableau 2). Le désir d'enfant était aussi deux fois plus fréquent chez les femmes sans enfant que chez celles qui étaient déjà mères $(\mathrm{OR}=2,64$; IC $95 \%[1,67-4,16])$. II était également fortement lié au pays de naissance des femmes: deux fois plus élevé $(O R=2,38$; IC $95 \%[1,28-4,43])$ chez les femmes nées en Afrique sub-saharienne et six fois plus élevé $(\mathrm{OR}=5,73$; IC $95 \%$ [1,74-18,85]) chez les femmes nées en Afrique du Nord que chez celles nées en Europe.

La relation de couple influait également sur le désir d'enfant. Les femmes qui n'avaient pas eu de partenaire sexuel dans l'année précédente, celles qui n'avaient que des partenaires occasionnels et celles dont le partenaire était séropositif pour le VIH rapportaient un désir d'enfant moindre que celles qui avaient un seul partenaire, celui-ci étant séronégatif ( $O R=0,22$; IC $95 \%[0,15$ $0,27] ; \mathrm{OR}=0,40$; IC $95 \%[0,22-0,74]$ et $\mathrm{OR}=0,48$; IC $95 \%$ $[0,26-0,87]$, respectivement).

À la question de la perception qu'elles avaient du risque de transmission du VIH à leur enfant pendant la grossesse et l'accouchement, seulement un tiers ( $33 \%$ ) pensait que celui-ci était faible ou très faible. Cette perception était corrélée au désir d'enfant. Ainsi, $26 \%$ des femmes considérant qu'il était très élevé et $43 \%$ de celles, qui pensaient qu'il était très faible, exprimaient le désir d'avoir un enfant.

En France, depuis 2001, les femmes séropositives souffrant d'infertilité peuvent avoir recours à l'assistance médicale à la procréation. Plus d'une sur deux ( $55 \%$ ) disait être au courant de cette possibilité et, parmi elles, plus de $50 \%$ déclaraient qu'elles y auraient recours en cas de besoin.

\section{Discussion}

Le désir d'enfant chez les personnes vivant avec le VIH/sida est peu documenté. Cette étude montre que $33 \%$ des femmes séropositives pour le VIH vivant en France en 2003 et en âge d'avoir des enfants souhaitent être mères. Les femmes qui veulent des enfants sont plus jeunes et vivent plus souvent en couple avec un partenaire séronégatif pour le VIH que celles qui n'en veulent pas. Leur état de santé par rapport au VIH ne semble pas lié au projet d'enfant. Dans une étude similaire réalisée aux États-Unis en 2000, le pourcentage de femmes séropositives souhaitant avoir des enfants était équivalent [1]. Des données françaises, recueillies en population générale sur la fertilité envisagée, ont été publiées en 2005 [7]. Elles font état d'un désir d'enfant, tel que nous l'avons défini ici, de $46 \%$, proportion franchement plus élevée que dans notre étude.

Le désir d'enfant n'était pas homogène parmi les femmes séropositives pour le $\mathrm{VIH}$ en âge de procréer. II varie beaucoup en fonction du pays de naissance : il est très important chez les femmes nées en Afrique. Par rapport aux femmes nées en Europe, les femmes d'Afrique du Nord expriment six fois plus souvent un désir d'enfant et les femmes d'Afrique sub-saharienne deux fois plus souvent. Cela reflète très probablement des différences culturelles du modèle de fécondité, ainsi que de la perception des barrières à la parentalité. Le désir d'enfant était également différent en fonction du fait d'avoir ou non déjà un enfant. Les femmes qui n'étaient pas encore mères avaient un désir d'enfant plus important que les femmes ayant des d'enfants. Cela est peutêtre à rapprocher des normes sociales dominantes qui veulent que la reproduction (ou la maternité) fasse partie intégrante de la vie des femmes [8].

Ces résultats confirment ceux des études antérieures montrant que l'état de santé par rapport au VIH n'est pas un facteur décisif 
Tableau 2

Analyses univariée (première colonne) et multivariée (deuxième colonne) des facteurs associés au désir d'enfant $(n=555)$

\begin{tabular}{|c|c|c|c|}
\hline & $\begin{array}{l}\text { \% de désir d'enfant } \\
\text { (Test de Wald) }\end{array}$ & $\begin{array}{c}\text { OR } \\
\text { ajusté }\end{array}$ & IC $95 \%$ \\
\hline $\begin{array}{l}\text { Année de diagnostic } \\
1980-1987 \\
1988-1990 \\
1991-1993 \\
1994-1996 \\
1997-1999 \\
2000-2003 \\
\end{array}$ & 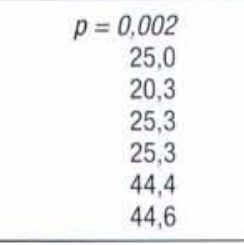 & $\begin{array}{r}1 \\
0,69 \\
0,80 \\
0,68 \\
1,14 \\
0,93\end{array}$ & $\begin{array}{l}(0,28-1,72) \\
(0,31-2,04) \\
(0,24-1,93) \\
(0,43-3,07) \\
(0,32-2,67)\end{array}$ \\
\hline $\begin{array}{l}\text { Âge } \\
<30 \text { ans } \\
30-34 \text { ans } \\
35-39 \text { ans } \\
40-44 \text { ans }\end{array}$ & $\begin{array}{r}p<0,001 \\
54,2 \\
37,7 \\
34,9 \\
14,9\end{array}$ & $\begin{array}{r}2,01 \\
1 \\
1,32 \\
0,38\end{array}$ & $\begin{array}{r}(1,03-3,92)^{*} \\
(0,72-2,43) \\
(0,19-0,77)^{*}\end{array}$ \\
\hline $\begin{array}{l}\text { Activité } \\
\text { Emploi } \\
\text { Chômage } \\
\text { Inactivité }\end{array}$ & 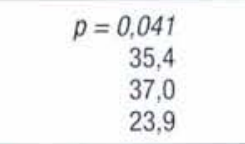 & $\begin{array}{r}1 \\
1,02 \\
0,59\end{array}$ & $\begin{array}{r}(0,59-1,76) \\
(0,33-1,04) \dagger\end{array}$ \\
\hline $\begin{array}{l}\text { Niveau d'études } \\
\text { Collège } \\
\text { CAP, BEP } \\
\text { Niveau lycée } \\
\text { Cycle supérieur } \\
\end{array}$ & 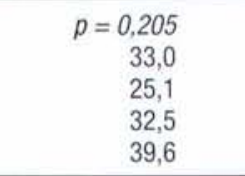 & $\begin{array}{r}1 \\
0,86 \\
0,94 \\
1,22\end{array}$ & $\begin{array}{l}(0,44-1,66) \\
(0,53-1,68) \\
(0,62-2,42)\end{array}$ \\
\hline $\begin{array}{l}\text { A des enfants } \\
\text { Oui } \\
\text { Non }\end{array}$ & $\begin{array}{r}p<0,001 \\
24,4 \\
39,2\end{array}$ & $\begin{array}{r}1 \\
2,64\end{array}$ & $(1,67-4,16)^{\ldots \ldots}$ \\
\hline $\begin{array}{l}\text { Situation de couple } \\
\text { Pas d'activité sexuelle dans l'année } \\
\text { Couple séroconcordant }{ }^{1} \\
\text { Couple sérodiscordant exclusif }{ }^{2} \\
\text { Couple sérodiscordant non exclusif }{ }^{3} \\
\text { Partenaires occasionnels uniquement }\end{array}$ & 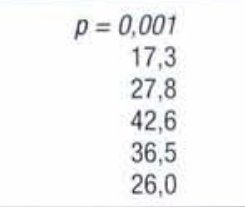 & $\begin{array}{r}0,22 \\
0,48 \\
1 \\
0,69 \\
0,40\end{array}$ & $\begin{array}{r}(0,10-0,47)^{* * *} \\
(0,26-0,87)^{*} \\
(0,29-1,67) \\
(0,22-0,74)^{\star *}\end{array}$ \\
\hline $\begin{array}{l}\text { Pays de naissance } \\
\text { France/Europe } \\
\text { Afrique sub-saharienne } \\
\text { Maghreb } \\
\text { Autres }\end{array}$ & $\begin{array}{r}p<0,001 \\
25,1 \\
47,1 \\
55,0 \\
18,1\end{array}$ & $\begin{array}{r}1 \\
2,38 \\
5,73 \\
0,56\end{array}$ & $\begin{array}{r}(1,28-4,43)^{* *} \\
(1,74-18,85)^{* *} \\
(0,12-2,64)\end{array}$ \\
\hline $\begin{array}{l}\text { Toxicomanie intraveineuse } \\
\text { Oui } \\
\text { Non }\end{array}$ & $\begin{array}{r}p=0,006 \\
20,9 \\
35,5\end{array}$ & $\begin{array}{r}0,83 \\
1\end{array}$ & $(0,40-1,71)$ \\
\hline $\begin{array}{l}\text { Stade sida } \\
\text { Oui } \\
\text { Non }\end{array}$ & $\begin{array}{r}p=0,508 \\
29,2 \\
33,2\end{array}$ & $\begin{array}{r}1,13 \\
1\end{array}$ & $(0,59-2,14)$ \\
\hline $\begin{array}{l}\text { Sous traitement antirétroviral } \\
\text { Oui } \\
\text { Non }\end{array}$ & 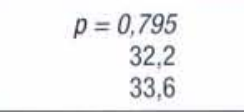 & $\begin{array}{r}1 \\
0,73\end{array}$ & $(0,39-1,36)$ \\
\hline $\begin{array}{l}\text { Bon contrôle de l'infection à VIH } \\
\text { Oui } \\
\text { Non }\end{array}$ & $\begin{array}{r}p=0,677 \\
31,8 \\
33,6\end{array}$ & $\begin{array}{r}1 \\
1,15\end{array}$ & $(0,70-1,89)$ \\
\hline
\end{tabular}

t-test: ***$p \leqslant 0,001 ;{ }^{* *} p \leqslant 0,01 ;{ }^{*} p \leqslant 0,05 ; \dagger p \leqslant 0,10$.

1 Partenaire masculin séropositif pour le VIH, sans partenaires occasionnels $;{ }^{2}$ partenaire masculin séronégatif pour le VIH, sans partenaires occasionnels : ${ }^{3}$ partenaire masculin séronégatif pour le VIH, avec partenaires occasionnels. 
dans l'expression du désir d'enfant [9]. Dans une étude publiée en 1995, c'est-à-dire avant l'accès aux trithérapies et à une période ou la morbidité et la mortalité liées au sida étaient très élevées, il avait été montré que la gravité des problèmes de santé liés au VIH n'interférait pas avec le désir exprimé par les femmes séropositives d'être mères [9]. La même observation est enregistrée à l'ère des thérapies antirétrovirales hautement actives, à partir de l'enquête Vespa. Que les femmes soient séropositives ou au stade sida, qu'elles soient ou non sous trithérapie, que la maladie soit bien contrôlée ou non, n'influent pas sur leur expression d'un désir d'enfant. En revanche, la situation du couple et, au sein du couple, le statut du partenaire par rapport au VIH, sont des facteurs qui interviennent fortement sur le désir d'enfant. Si le partenaire est séropositif, le désir d'enfant est deux fois moins fréquent que s'il est séronégatif où de statut non connu par la femme. D'autres études ont également montré l'importance qu'avait le statut VIH du partenaire dans le désir d'enfant $[1,10]$. Chez les femmes vivant avec le VIH/sida, il semble donc que ce soient les déterminants classiques qui gouvernent le désir d'enfant, sans que l'infection par le VIH elle-même participe de ce désir dès lors que le futur père n'est pas infecté [11].

Maintenant qu'elles sont informées que le risque de transmission du virus à l'enfant est bien contrôlé, il est probable que dans un avenir proche, de plus en plus de femmes séropositives vont désirer être enceintes [12]. L'évolution des caractéristiques démographiques de l'épidémie féminine en France, avec une augmentation du nombre de jeunes femmes d'origine sub-saharienne, va probablement contribuer à l'augmentation du nombre de grossesses chez les femmes séropositives. De plus, l'accès dorénavant possible à l'assistance médicale à la procréation médicalement assistée en cas de risque viral permet d'obtenir des grossesses sans risque de contamination entre les futurs parents [13]. Cette possibilité pourrait aussi contribuer à l'augmentation des grossesses chez les couples vivant avec le VIH/ sida.

II devient ainsi important d'inclure dans la prise en charge des femmes et des hommes séropositifs un volet dédié à la reproduction. Celui-ci devrait proposer un accès à une contraception efficace si elle est souhaitée, mais également des informations qui permettent une conception sans risque de contamination ni entre les parents ni des enfants.

\section{Références bibliographiques}

1. Chen J, Phillips K, Kanouse D, Collins R, Miu A. Fertility desires and intentions of HIV-positive men and women. Fam Plann Perspect $2001 ; 33$ : 144-152.

2. Saada M, Le Chenadec J, Berrebi A, et al. Pregnancy and progression to AIDS : results of the French prospective cohorts. SEROGEST and SEROCO Study Groups. Aids $2000 ; 14: 2355-2360$.

3. Nebie Y, Meda N, Leroy V, et al. Sexual and reproductive life of women informed of their HIV seropositivity : a prospective cohort study in Burkina Faso. J Acquir Immune Defic Syndr $2001 ; 28: 367-372$.

4. Mocroft A, Ledergerber B, Katlama C, et al. Decline in the AIDS and death rates in the EuroSIDA study : an observational study. Lancet $2003 ; 362: 22-29$.

5. Mofenson LM. Advances in the prevention of vertical transmission of human immunodeficiency virus. Semin Pediatr Infect Dis 2003 ; 14 : 295-308.

6. Dray-Spira R, Spire B, Heard I. Heterogeneous response to HAART across a diverse population of HIV-infected individuals-Results from the Anrs-EN-12Vespa Study. AIDS 2006 ; 21 suppl 1 : S5-S12.

7. Toulemon L, Testa M. Fécondité envisagée, fécondité réalisée : un lien complexe. Population \& Sociétés $2005 ; 415: 1-4$.

8. Sowell RL, Misener TR. Decisions to have a baby by HIV-infected women. West J Nurs Res 1997 ; 19: 56-70.

9. Kline A, Strickler J, Kempf J. Factors associated with pregnancy and pregnancy resolution in HIV seropositive women. Soc Sci Med 1995; 40 : 1539-47.

10. Heard I, Potard V, Costagliola D, Kazatchkine MD. Contraceptive use in HIVpositive women. J Acquir Immune Defic Syndr $2004 ; 36: 714-720$.

11. Sowell RL, Murdaugh CL, Addy CL, Moneyham L, Tavokoli A. Factors influencing intent to get pregnant in HIV-infected women living in the southern USA. AIDS Care 2002; $14: 181-191$.

12. Kirshenbaum SB, Hirky AE, Correale J, et al, « Throwing the dice » : pregnancy decision-making among HIV-positive women in four U.S. cities. Perspect Sex Reprod Health $2004 ; 36$ : 106-113.

13. Kim L, Johnson M, Barton S, et al. Evaluation of sperm washing as a potential method of reducing HIV transmission in HIV-discordant couples wishing to have children. AIDS. $1999 ; 13: 645-651$ 